\title{
PIV measurements of airflow past multiple cylinders
}

\author{
Waldemar Wodziak', ${ }^{1}$ Jacek Sobczyk ${ }^{1}$ \\ The Strata Mechanics Research Institute of The Polish Academy of Sciences, Reymonta 27, 30-059 Krakow, Poland
}

\begin{abstract}
Flow characteristics in vicinity of six circular cylinders aligned inline was investigated experimentally by means of PIV method. Experiments were conducted in a low speed closed circuit wind tunnel. Inflow velocity was $1.2 \mathrm{~m} / \mathrm{s}$ which corresponds to $R e=1600$ based on the cylinder diameter. Spacing ratio between cylinders $L / D$ was 1.5 . Instantaneous and averaged velocity fields were presented. Experiments were designed in order to use their results as a test case for future numerical calculations.
\end{abstract}

\section{Introduction}

Fluid flow around cylinders is subject of interest theoretical and applied investigations. Knowledge about flow characteristic is essential for example to determine heat transfer intensity in rod structure of nuclear reactors or heat exchangers. Fluid flow over one and two cylinders has been studied extensively and is well known [1], [2], [3]. Depending on Reynolds number and distance between cylinders different flow patterns are observed.

Airflows in mining excavations are disturbed by presence of various devices. Velocity field changes and separation and recirculation zones may appear. Support roof system is widely used in mining longwalls for ceiling protection. One of the components of support roof system are legs which are approximately cylindershaped and distributed inline. This configuration may be considered as flow through multiple cylinders.

Configuration of multiple array of cylinders has been discussed in several works. Effect of gap spacing on vortex shedding of laminar flow past six cylinders arranged in-line are studied by [4] and [5]. They found a flow pattern transition between spacing ratios 3.6 and 4 when drag and lift coefficients change significantly. Studies of flow around six cylinders are mostly based on CFD calculations and only for low Reynolds numbers. However, the main limitation of computer modelling is the need to verify the applied numerical models, typically by experimental testing [6],[7].

Experimental results of turbulent airflow through six cylinders presented in this paper can be used as a benchmark for future CFD validation.

\section{Experimental setup}

Measurements were performed a in low speed closedcircuit wind tunnel. Total dimensions of wind tunnel are $9,79 \times 4,08 \times 2,34 \mathrm{~m}$. A $30 \mathrm{~kW}$ fan is used to create a desired flow in the test section. Velocity range is from 0.1 to $60 \mathrm{~m} / \mathrm{s}$ and longitudinal free stream turbulent intensity is less than $0.5 \%$. Wind tunnel is equipped with cooling system to control temperature and humidity. Test section have dimensions: a length of $1.5 \mathrm{~m}$, height and width $0.5 \mathrm{~m}$ and is adapted to Stereo PIV measurements [8].

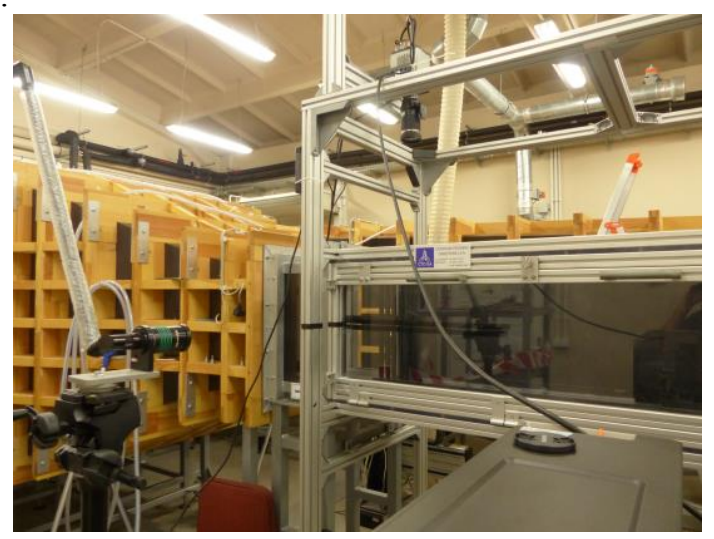

Fig. 1. Experimental setup

Particle image velocimetry is a non-intrusive method used to obtain velocity fields. Particle markers are introduced to measured area and illuminated twice by laser sheet. Camera placed commonly perpendicularly to illuminated plane records two images. Particles on the first frame are distributed stochastically. Next, this pattern is found on second frame. Particle displacement is calculated by means of statistical methods. Time between laser pulses is known therefore velocity vector can be obtained [9].

The seeding for the PIV method was DEHS oil sprayed through a seeding generator. The tested planes were illuminated by a two-impulse Nd:YAG laser and particle images were registered by two sCMOS cameras with a 5,5 MPx resolution. Each time 300 images were recorded with the frequency $15 \mathrm{~Hz}$. Calibration was carried out before measurements. Adaptive correlation with $32 \times 32 \mathrm{px}$ interrogation window was used to

* Corresponding author: wodziak@img-pan.krakow.pl 
calculate velocity fields. The free-stream velocity was measured using a Schmidt anemometer.

Velocity range for airflow through legs in mining excavations is from 0.1 to $20 \mathrm{~m} / \mathrm{s}$ which corresponds to Re number (based on leg diameter $D=0.2 \mathrm{~m}$ ) from 1300 to 270000. In wind tunnel experiments inflow velocity was $V_{0}=1.2 \mathrm{~m} / \mathrm{s}$ which gives a Reynolds number about 1600 (based on the cylinder diameter). 2D measurements were conducted for constant temperature $\left(21^{\circ} \mathrm{C}\right)$, humidity $(\varphi=64,9 \%)$ and atmospheric pressure $(980$ $\mathrm{hPa}$ ). Spacing between cylinder was $L / D=1.5$. Measured areas were located in lateral region and second on the wake region of airflow (Fig.2). Coordinate system origin is placed at the center of the upstream cylinder.

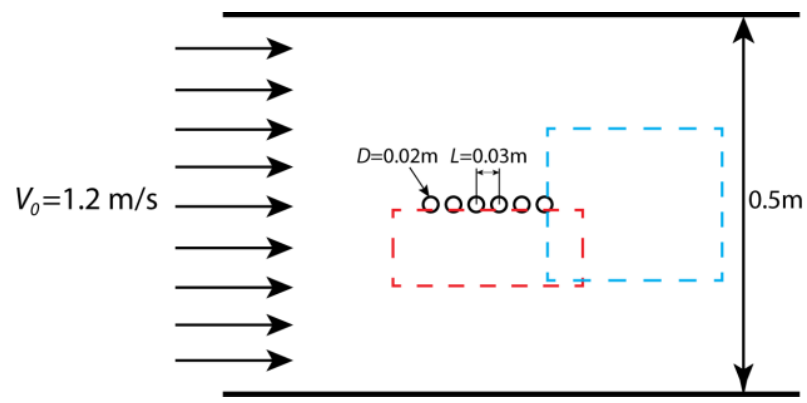

Fig. 2. Measured areas at test section

\section{Results of PIV measurements}

Normalized mean velocity components and their standard deviations contours calculated for lateral region are presented in Fig.3-6. Mean values are averaged over 300 images. There is an aerodynamic shadow between first two cylinders with virtually no mass exchange in the y direction. Following cylinders are more and more infected by vortices shed from the first one.

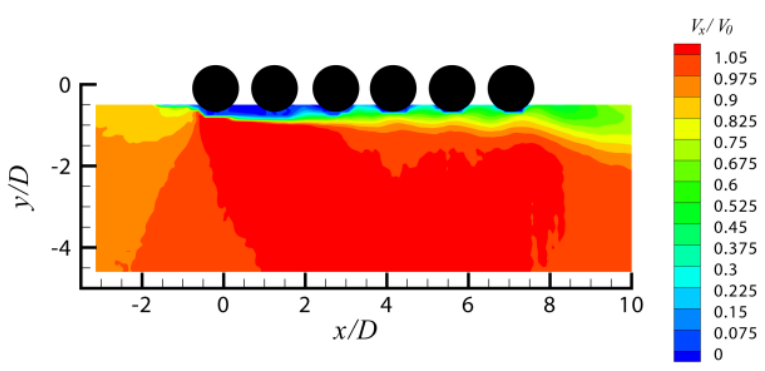

Fig. 3. Normalized mean streamwise velocity component contour plot obtained for lateral region

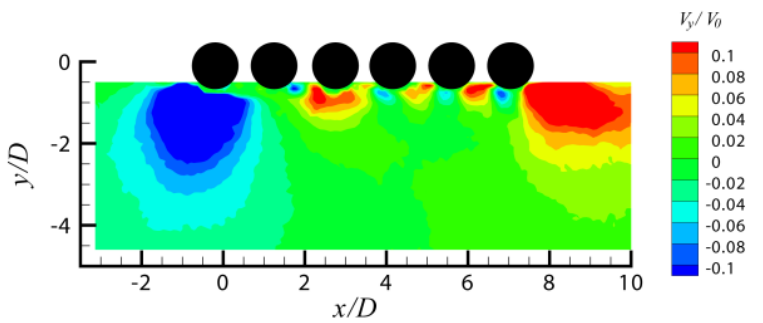

Fig. 4. Normalized mean transverse velocity component contour plot for obtained lateral region

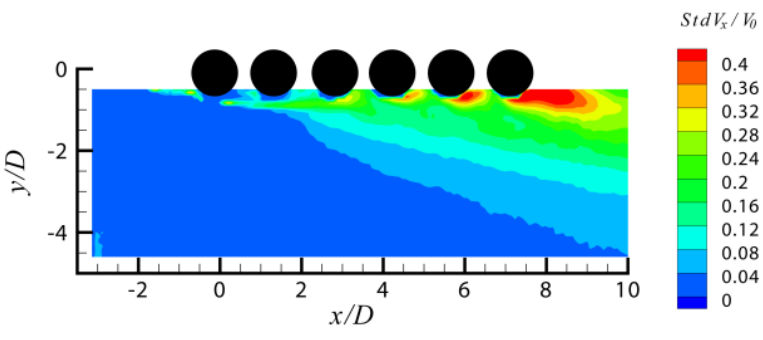

Fig. 5. Normalized mean standard deviation of streamwise velocity component contour plot for lateral region

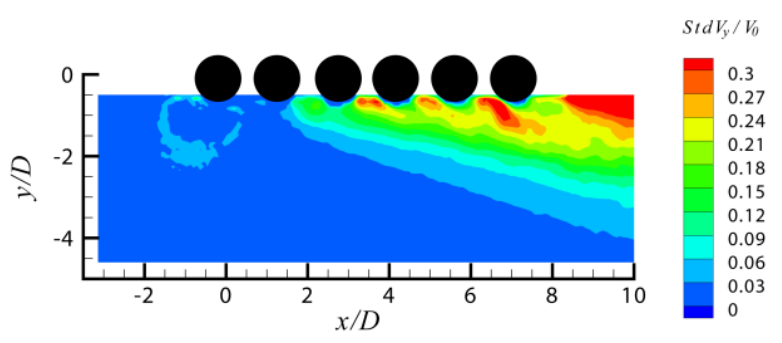

Fig. 6. Normalized mean standard deviation of transverse velocity component contour plot obtained for lateral region

PIV method provides instantaneous measurements of velocity vectors. Normalized velocity magnitude $V$ contours at four time steps obtained for lateral region are presented in Fig 7-10. For better visualization of vortices from instantaneous velocity fields free stream velocity $V_{0}$ was subtracted and streamlines on the basis of remaining information were plotted. Contour plots presented on Fig. 7-10 and 15-18 contain complete information about $V$.

Time step between frames was $\sim 0.06 \mathrm{~s}$. Vortex shed from the first cylinder moved along following cylinders trying to enter in between them to create wakes. Remaining cylinders interfered with the wake dynamics and vortex formation region of the first cylinder. Vortices slid over them to finally form von Karman street in the wake region.

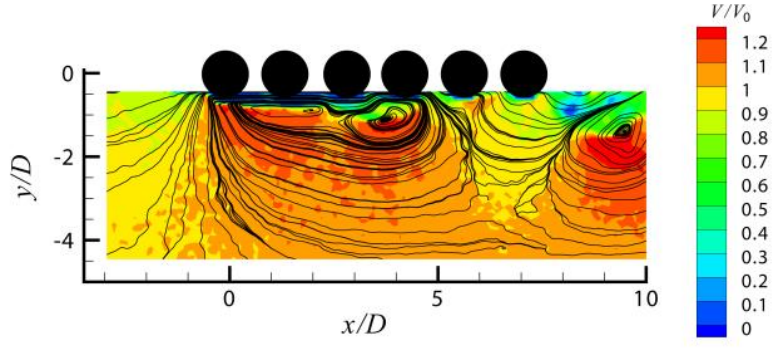

Fig. 7. Normalized velocity magnitude contour plot at first time step obtained for lateral region 


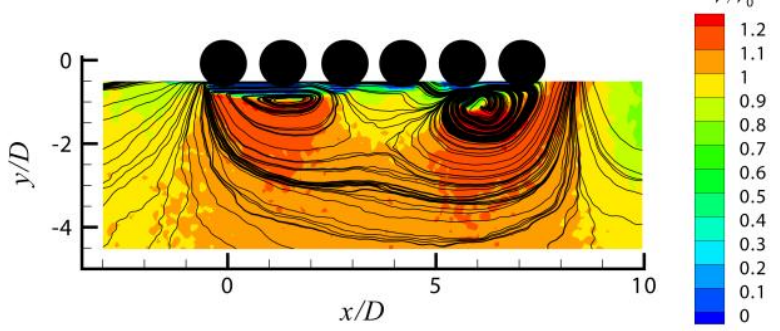

Fig. 8. Normalized velocity magnitude contour plot at second time step obtained for lateral region

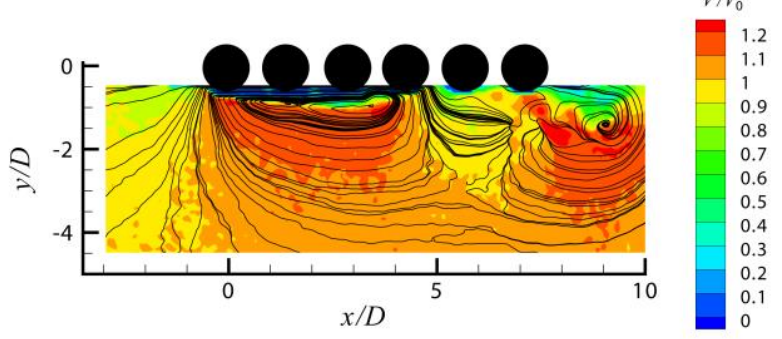

Fig. 9. Normalized velocity magnitude contours and third time step obtained for lateral region

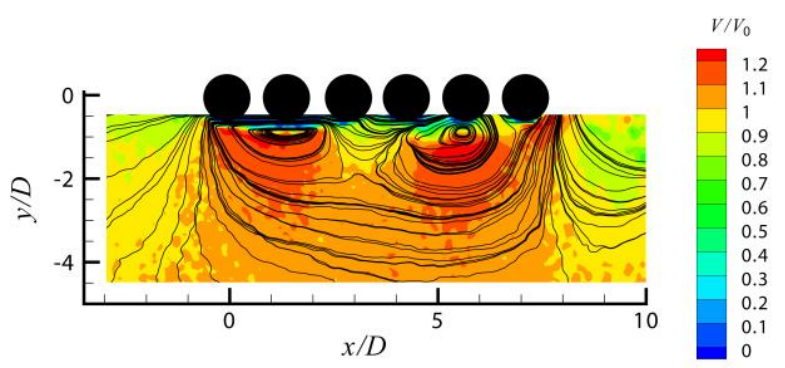

Fig. 10 Normalized velocity magnitude contours and fourth time step obtained for lateral region

Mean velocity components and their standard deviations are showed in Fig. 11-14.

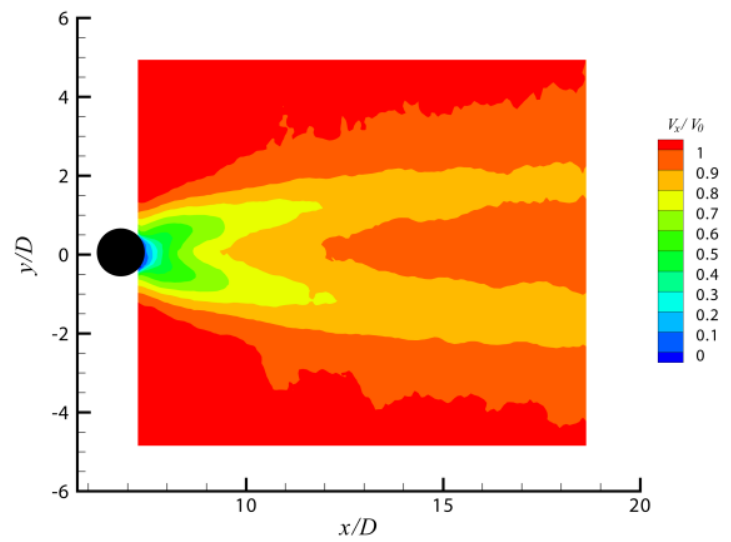

Fig. 11. Normalized mean streamwise velocity component contour plot obtained for wake region

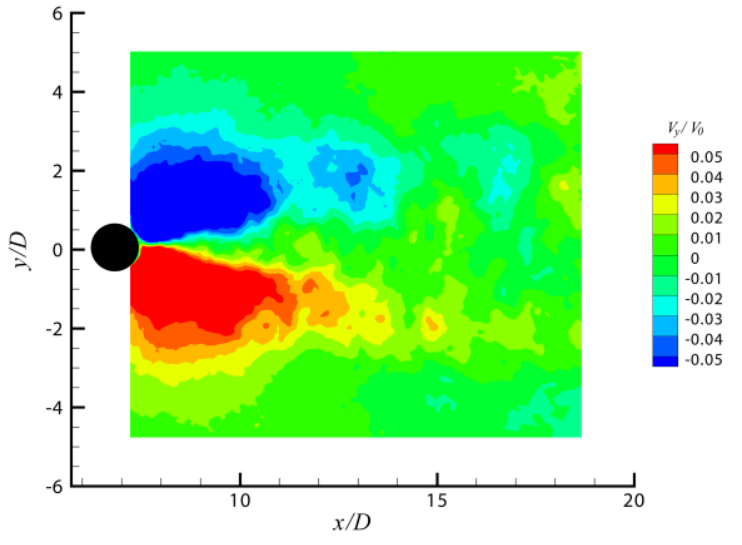

Fig. 12. Normalized mean transverse velocity component contour plot obtained for wake region

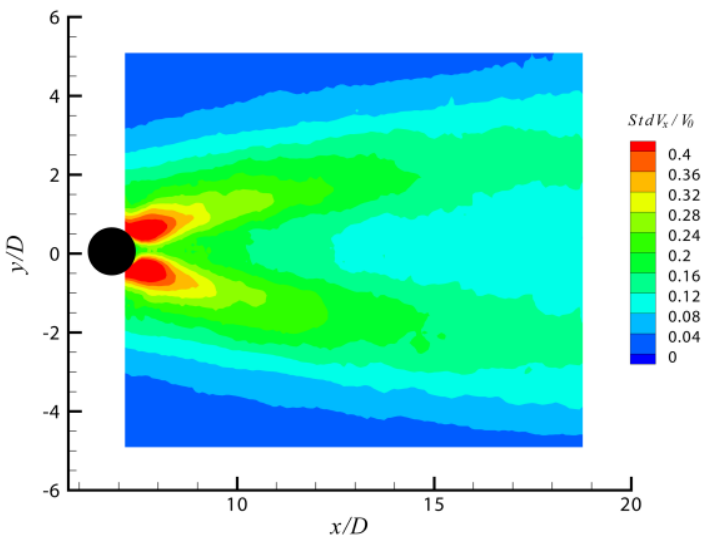

Fig. 13. Normalized mean standard deviation of streamwise velocity component contour plot obtained for wake region

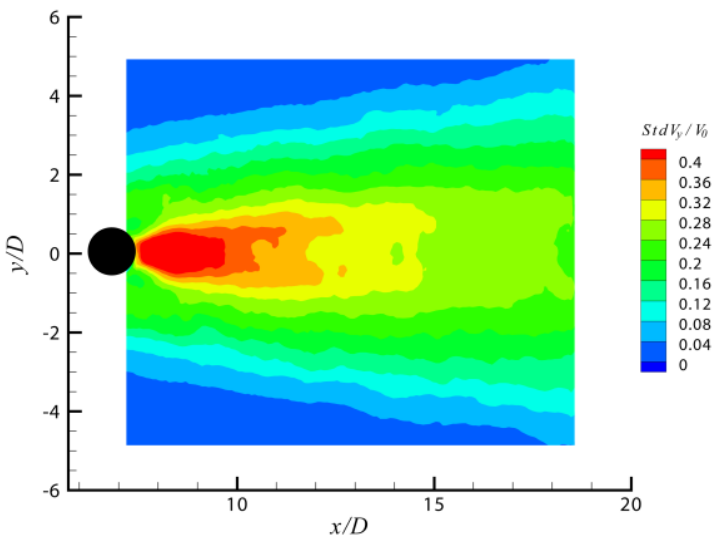

Fig. 14. Normalized mean standard deviation of transverse velocity component contour plot obtained for wake region

Normalized velocity value vector contours and vortex visualization at four time steps obtained for wake region are presented on Figures 15-18. Time difference between frames is $0.06 \mathrm{~s}$. Vortex shedding from the outer side of cylinders forms a single vortex street. 


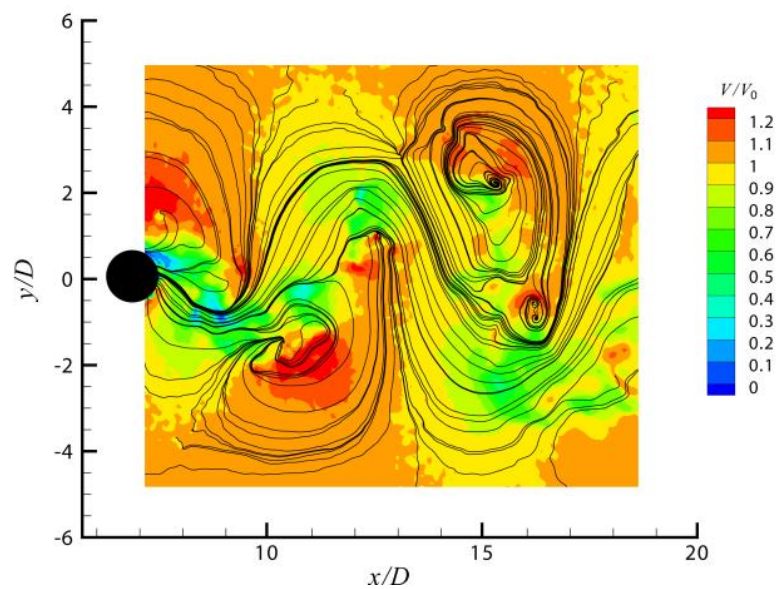

Fig.15. Normalized velocity magnitude contour plot at first time step obtained for wake region

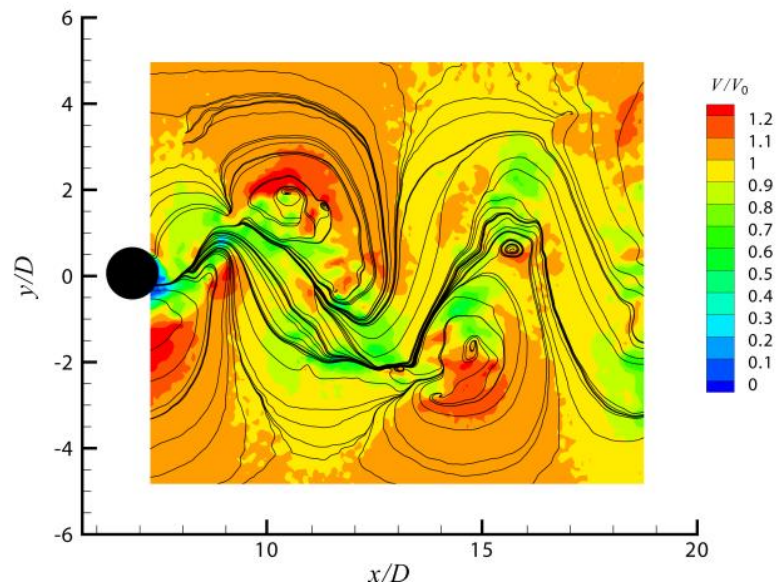

Fig.16. Normalized velocity magnitude contour plot at second time step obtained for wake region

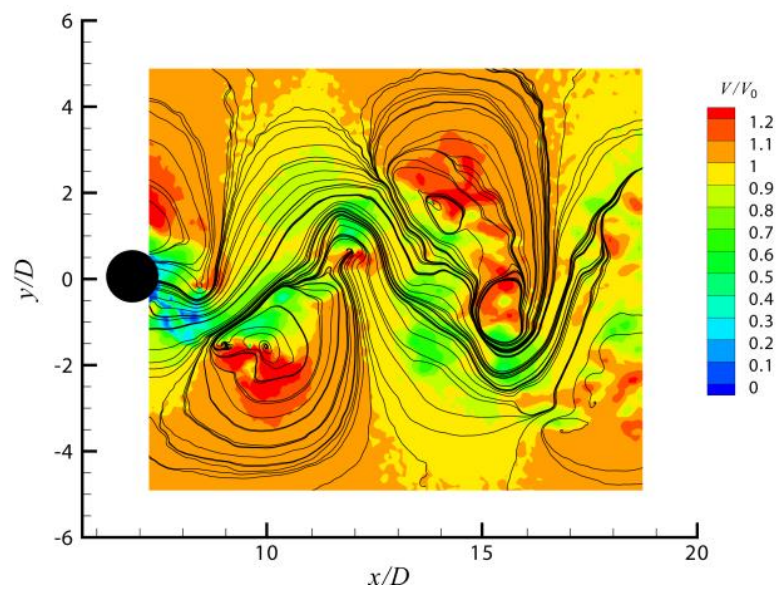

Fig.17. Normalized velocity magnitude contour plot at third time step obtained for wake region

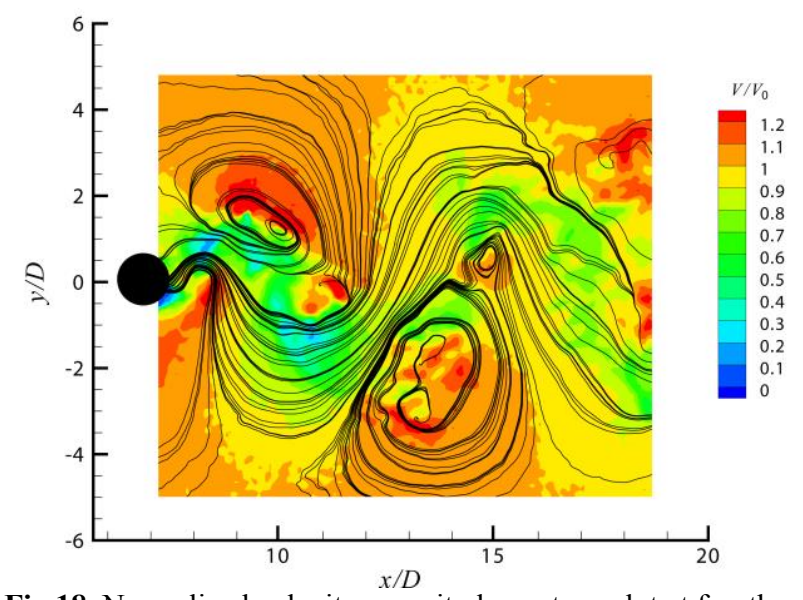

Fig.18. Normalized velocity magnitude contour plot at fourth time step obtained for wake region

\section{Conclusions}

Preliminary experimental results of airflow past six cylinders including mean and instantaneous velocity vector fields are presented. Flow past multiple cylinders is frequently seen in engineering. Knowledge about velocity fields in mining excavations is important in terms of proper ventilation. Cylinder-shaped legs modify velocity fields in the longwall area. In future works PIV results can be compared to CFD calculations.

\section{Acknowledgment}

This publication presents results of the work supported by the Grant for the Young Researchers the Polish Minstry of Science and Higher Education (7443/E-43/M/2017) and the Statutory Research of IMG-PAN

\section{References}

1. D. Sumner, J. Fluid Struct, 26, 849-899 (2010)

2. Y.Zhou, Md. Mahbub Alam, J. Heat and Fluid Flow, 62, 510-537, (2016)

3. C. Demartino, F. Ricciardelli, Eng. Struct, 137, 76114, (2017)

4. C. Liang, X. Luo, Comput. Fluids, 38, 950-964, (2009)

5. F. Fornarelii, P. Oresta, A. Lippolis, J. Heat Mass Trans., 11, 1-28, (2015)

6. J. Szmyd, M. Branny, M. Karch, W. Wodziak, M. Jaszczur, R. Nowak, Arch. Min. Sci., 58, 333-348, (2013)

7. M. Branny, M. Karch, W. Wodziak, M. Jaszczur, R. Nowak, J. Szmyd, J. Phys. Conf.. Ser., 530, 0120291-012029-8, (2014)

8. M. Bujalski, M. Gawor, J. Sobczyk, Transactions Of The Strata Mechanics Research Institute (in Polish), 15 (2013)

9. M. Raffel, C.E Willert, S. Wereley, J.Kompenhans, Particle Image Velocimetry. A Practical Guide, Springer, 2007 